
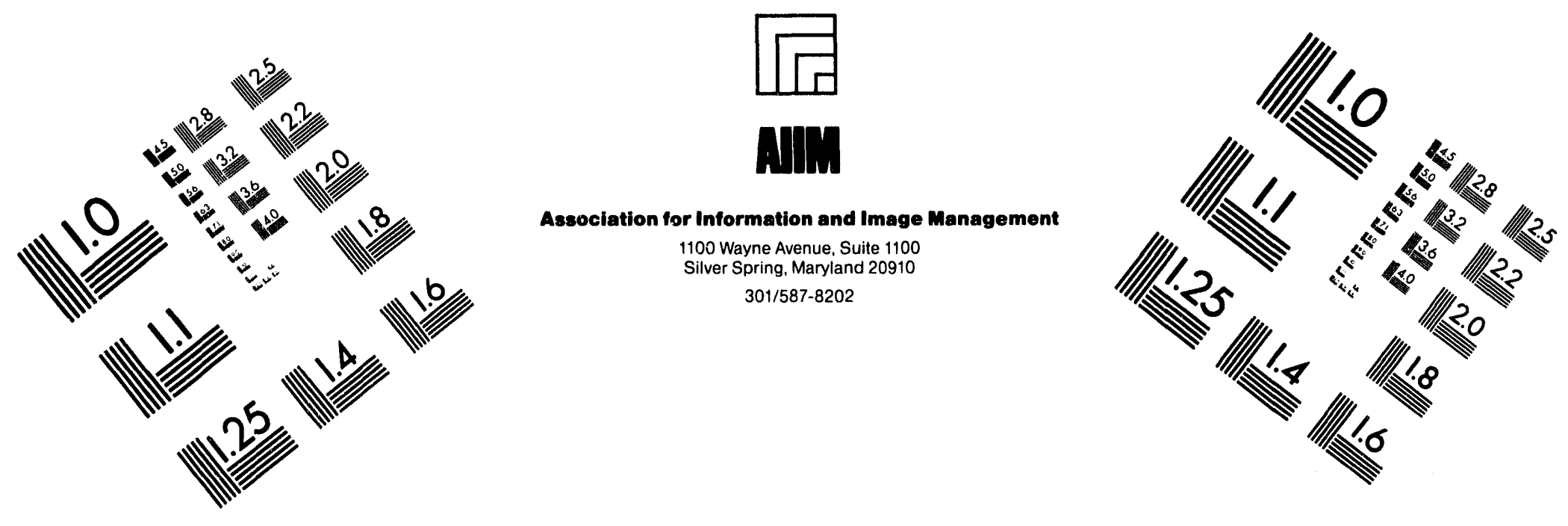

\title{
Centimeter
}

$\begin{array}{llllllllllllllll}1 & 2 & 3 & 4 & 5 & 6 & 7 & 8 & 9 & 10 & 11 & 12 & 13 & 14 & 15 & \mathrm{~mm}\end{array}$

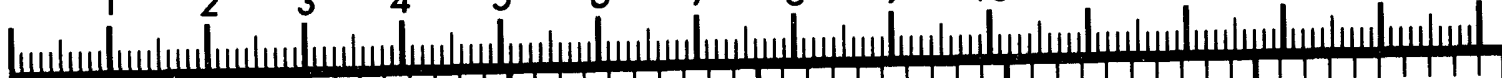

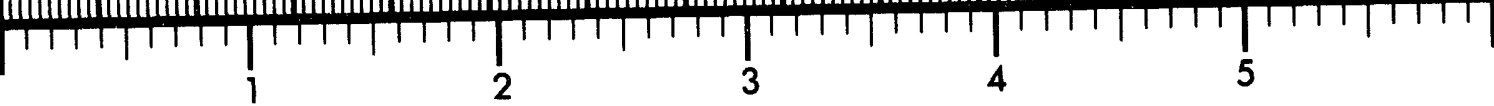
Inches
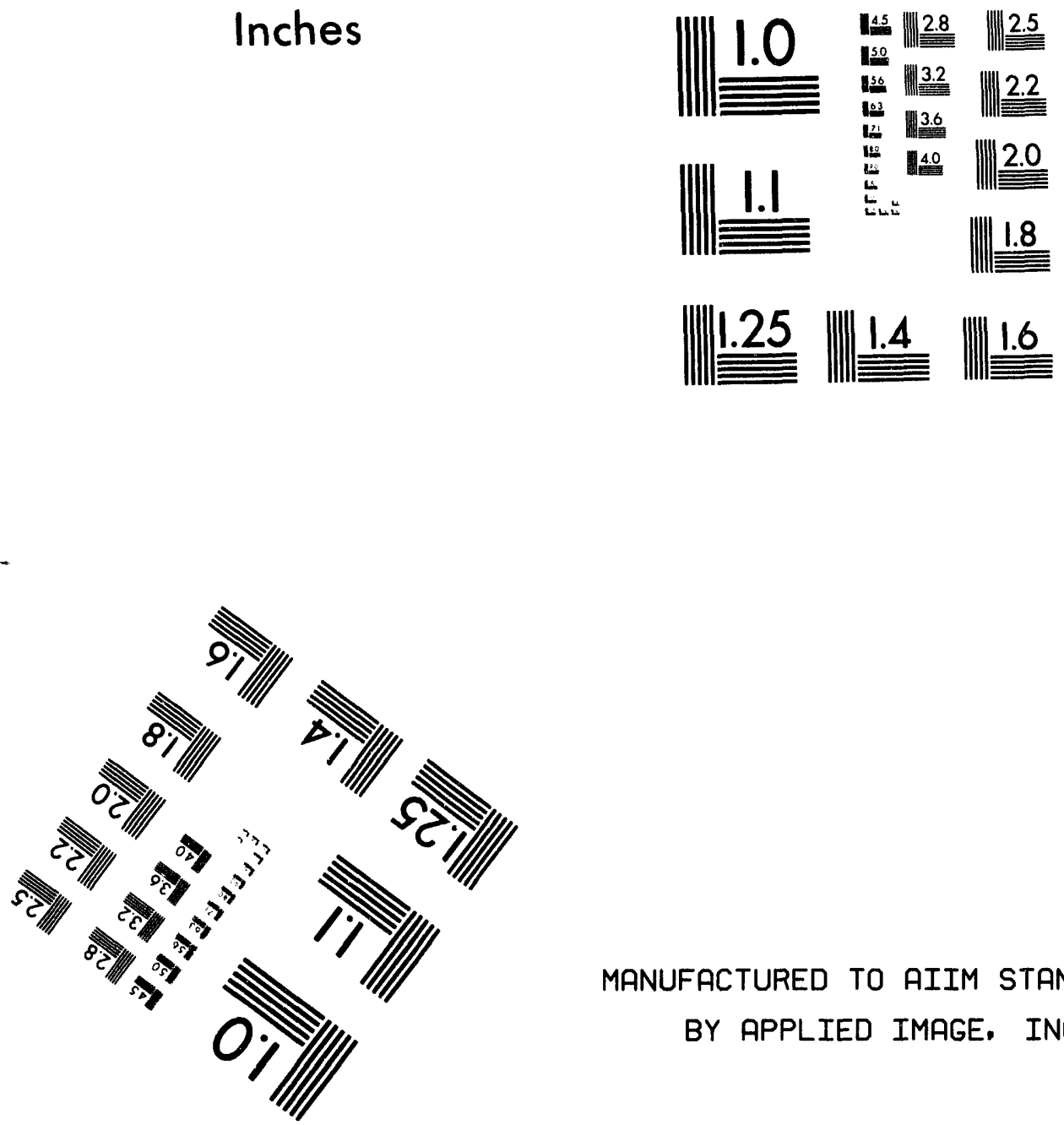

MANUFACTURED TO AIIM STANDARDS

BY APPLIED IMAGE. INC.

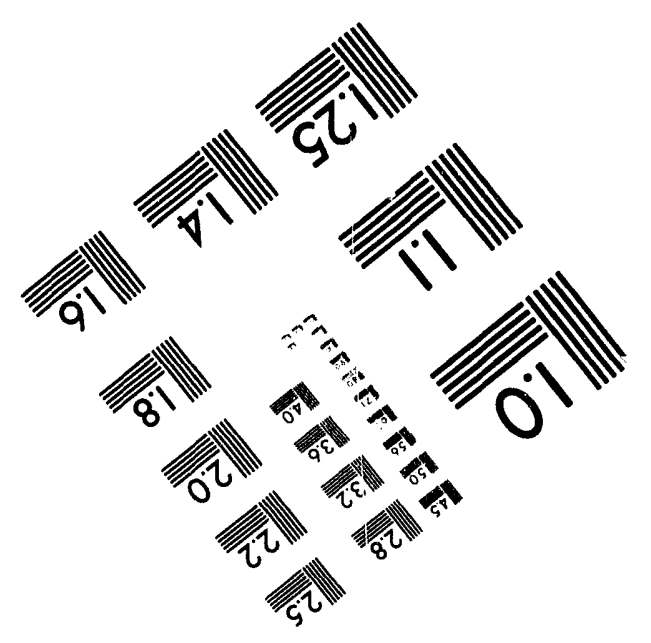



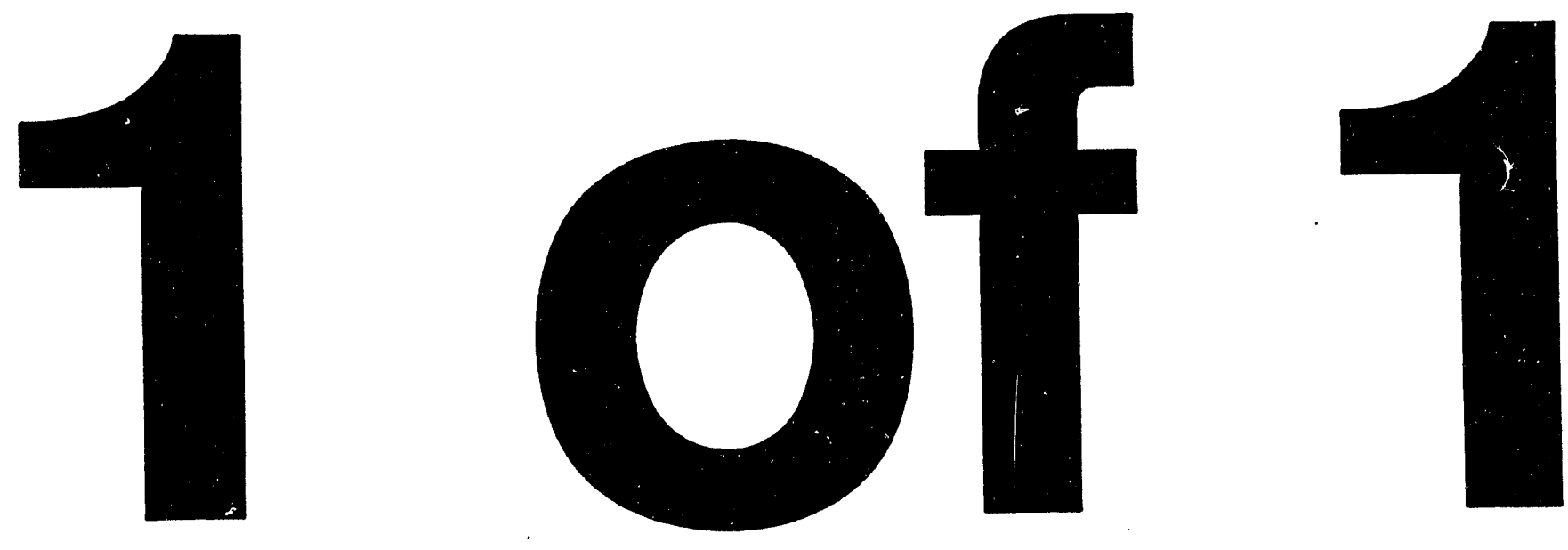
KCP-613-5288

Distribution Category UC-705

Approved for public release; distribution is unlimited.

\section{MOLECULAR MODEL GENERATOR TOOLKIT}

Richard D. Schneider

Published July 1994 


\section{What is in the package}

This user guide is provided alc.ng with the software. The software is an ASCll file of Fortran source code. Programs and subroutines must be compiled and a path must be set up to allow execution of the program. If it is necessary to modify the code for your application, keep a backup copy of both the original code and the modified code.

\section{What the software will do}

This software can help you create plastic models of molecules without having to buy, or train for, a CAD-CAM package. Most CAD systems on the market have problems trying to build complex molecules. Even if you have the expertise to build one on a CAD system, the time involved is substantial. With this software you can just enter seven parameters, and wait until the process is done.

This program reads a data file and generates output that will create a plastic model of the molecule described in the data file. The data file to be read must be from the Brookhaven Protein Databank ${ }^{1}$ or be in the same format as the files in the databank. Access to the databank is available with a modem, or a disk may be obtained from Brookhaven.

The model is created with overlapping spheres representing the atoms. The size of the spheres is calculated from the databank file such that each sphere will intersect at least one other sphere in the existing set. The amount of overlap is controlled by the program to allow sufficient rigidity of the model. There is an array (df) that varies the size of the atoms. Currently this is set to create visual difference between such things as phosphorus and oxygen. If you wish to make all the atoms the same size, the source file must be modified.

The output file is a program for a 3D Systems ${ }^{2}$ SLA (Stereolithography Apparatus). The user must specify what size of model is to be generated. This must be within the limits of the machine used by you or your vendor. ${ }^{3,4}$ The SLA 250, which is the most common, will create up to a 10 -inch cube.

\section{What hardware is required}

This program was run on an SGI Indigo workstation. It should work on most computers that have a Fortran compiler. The files generated are large and it is recommended that you have at least $100 \mathrm{mb}$ of available disk space to store the output files. For a large model, the file size could possibly be $100 \mathrm{mb}$ or larger. The output files are relatively large and will probably not fit on floppy disks. To transfer them to a vendor will require a $4 \mathrm{~mm}$ dat tape or other means of transferring large files. The files can easily be 20 megabytes or larger. You should check with the vendor ahead of time to determine what media he can process.

\section{Using the toolkit}

Once you have the program compiled and ready to run and have the data files accessible, you need to create a parameter file to control the program's output.

The name of the input file and the parameters that you wish to use need to be in a file named sla.parm in your active directory. 
The sla.parm file must have seven lines with the following information.

1. The input flle name (or path) is the name of the file that the program will read as input. This is a file from Brookhaven Protein Databank. The program will generate a sphere for every line that starts with "ATOM" or "HETATM".

2. The pipe file name is the name of the file that you wish to hold the coordinates of the alpha-carbons. These coordinates will be scaled, rotated, and translated as crquired to fit the molecule into the cube specified. The file is formatted such that it can be read by Pro/ENGINEER ${ }^{5}$ as a datum point array. This array is then used to create a pipe. This can be converted to an .stl file and built on the SLA.

3. The "BALL" flle name is the name of the file that you wish to hold the coordinates of the "ATOM" and "HETATM". These coordinates will be scaled, rotated, and translated as required to fit the molecule into the cube specified. The range in $X$-axis is compared to that in $Y$ and $Z$. The smallest range is used as the $Z$-axis on the SLA machine. This is done to minimize build time on the machine. The radius of the spheres generated is calculated as the minimum that allows all atoms to intersect. If the file defines multiple chains, the radius calculated will be much too large and the model will be incorrect. See usage notes. "HETATM" molecules are generated as spheres the same size as atoms. If you don't want the "HETATM" s used, make a copy of the databank file and modify it by changing the string "HETATM" to "IGNORE".

4. The CUBE SIZE is the length of one edge of a cube that would enclose the model. The value entered is used to scale the databank coordinates to the desired model size.

5. The LAYER HT. is the thickness of layers created by the SLA. The normal range is between .005 and .012 . See usage notes.

6. The BALL RADIUS allows generation of multiple chains which do not intersect. If a value is entered, the program will use that value and not calculate a radius. The radius that you input is sized in relation to the databank file, not the model that will be built. Something between .95 and 1.0 is normal.

7. The LOWEST $Z$ is the bottom of the lowest ball created in the SLA. For example, if all that is desired is a model of the spheres (ATOMS), the normal start position would be .30. However, if there are two or more chains to be built at one time and the chains do not intersect, the start point may be .6 or higher to allow insertion of a base to hold the chains in relation to each other. This base would be an .stl file defined in Pro/ENGINEER, or whatever CAD system is available. 
For example the sla.parm file might look like this:

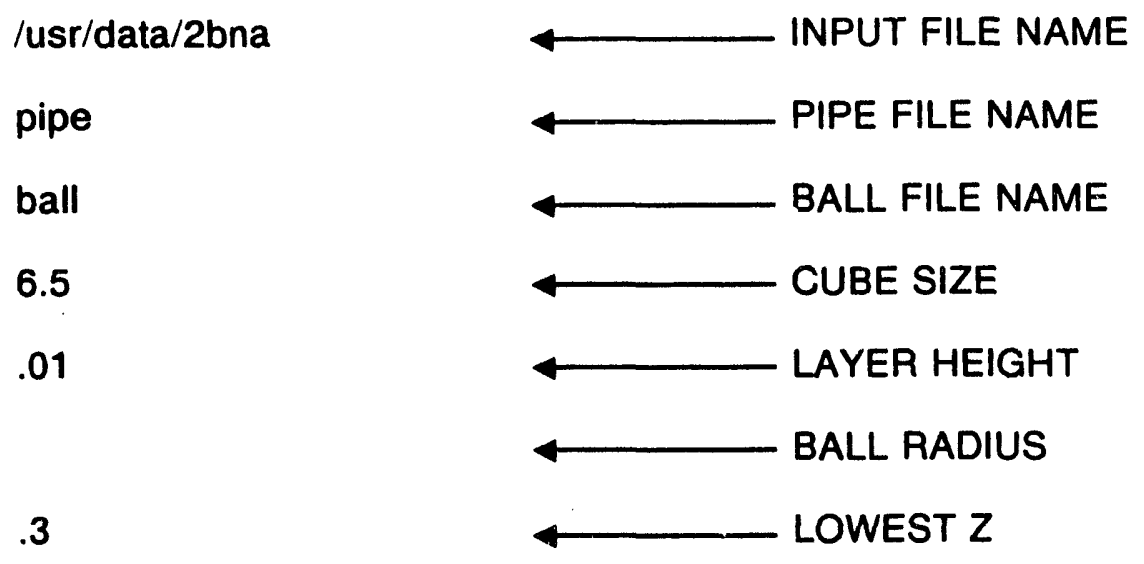

After creating the sla.parm file, you execute the program. When the program is done, there will be output files as follows.
1. bas.sli
is the slice file for the model's support
2. sli.sli
is the slice file for the model
3. "????"
the file you named on line 2 of the sla.parm file
4. "????"
the file you named on line 3 of the sla.parm file
5. sli.info
contains info about the model you have generated
6. sli.hdr
contains first 6 lines of databank file

The bas.sli, sti.sli, balls.stl, and sup.stl files are ASCII files that must be processed by the software provided by 3D Systems with the SLA. If you do not have an SLA, these files must be sent to the vendor to process on his machine. The vendor must be supplied with the layer height and the size of the model. If you have an SLA, see the "Information for the SLA operator" section below.

For those without an SLA, there are two vendors listed on the reference page ${ }^{3,4}$ who use SLAs; also, 3D Systems will do some custom pieces.

\section{Usage notes}

A modified copy of the databank file may be used as input to check the output. For instance, if multiple chains are in a single file, you may wish to delete all but a single chain and run the program on that file. Then use the radius value that the program calculated (this is in the sli.info file as original rad.) in line 5 of sla.parm and run the program on the original databank file to generate the output file.

Likewise, a modified copy of the databank file may be used to generate a model. For instance, with file 2 bna the HETATMs are water molecules. If you don't want to build 
these as part of the model, just delete them from the file or change HETATM to IGNORE, before running this program. If a file contains multiple chains and is processed without setting the radius parameter, the info file will contain error messages saying "intersection array counter is too big". If there are two or three of these messages the model may be ok, but if there are dozens or hundreds there is something seriously wrong.

The program checks on the second character of the two-character column that designates the atom type. The current values are designed to allow visual difference between the atoms. With some minor modifications, the array values could be changed to the Van der Waals radii or whatever values you feel are appropriate. The values in this array (df) are used as scale factors; therefore, the smallest value should be 1.00 . This is located in the source of subroutine fetch.

The ou'put has each successive coordinate set (sphere) connect to an existing set unless a radius value is entered. If a value is entered in line 6 of sla.parm, no checking will be done. Beware that if a value is entered that is too small to intersect the other spheres, the model will collapse.

The layer height for the model should be between 0.005 and 0.012 . As the values become smaller, the model will be smoother. However, the smaller layer height will create more layers in a given size model, thereby increasing the size of the output file. For testing, to see whether a specific data file will process properly, or to check the output radius size, the layer ht. may be set to .10 or some similar value to reduce run time for the program. Be aware that the output with a layer ht. greater than .012 will not work on the SLA but is useful only for testing. Also note that "normal" for the SLA is .010 .

\section{Information for the SLA operator}

In subroutine hatch, the hatch is set at $.012 "$ for hatch and $.005 "$ for skin. This may be changed in the source code if necessary for a specific resin. The hatch is staggered such that each successive layer does not directly overlay the previous. This is to help eliminate bubbles in the model.

Generating the build file for the SLA and checking it is accomplished using the "partman" and "sliconv" software from 3D Systems on an SGI workstation.

Use sliconv to convert the two files to binary files:

sliconv -b sli.sli balls.sli

sliconv -b bas.sli sup.sli

The .stl files (sup.stl and balls.stl) are brought into partman and saved. These are dummy files to allow partman software to be used. They simply define a box to generate the upper and lower bounds for partman. To use partman you must have .stl files with the same names as the .sli files.

When a base is needed to support the model, it must be created in a CAD package such as Pro/ENGINEER and the .stl file must be brought into partman.

In the partman main window, hit the button labeled "prepare". In the pop-up window the message for the components with .sli files should say "ready". If not, you must go to a 
unix shell window and enter touch *.sli. This will change the modification date on the files. Prepare should combine the .sli files without trying to slice the dummy.stl files. Do not use the Prepare All button.

The sli files and the.$v$ file can be viewed inside the partman view program to determine whether the output is what you want. If a base for the model is to be built in the SLA machine at the same time as the model, the base should be created in Pro/ENGINEER and brought into partman before the prepare. 


\section{References}

1 Protein Data Bank, Chemistry Department, Building 555, Brookhaven National Laboratory, Upton, NY 11973 *

2 3D Systems, Inc., 26081 Avenue Hall, Valencia, CA 91355-9935. *

${ }^{3}$ Laser Prototypes, Inc., 3155 Route 10, Denville, NJ 07834 *

${ }^{4}$ Prototype Express, Inc., 415 West Golf Road, Suite 25, Arlington Heights, IL 60005 *

* Note: These vendors are referenced here because the author dealt with them. Other vendors with comparable offerings may exist.

${ }^{5}$ Pro/ENGINEER is a registered trademark of Parametric Technology Corporation, 128 Technology Drive, Waltham, MA 02154. Note: This was the CAD system used by the author. Other systems may work equally well. 

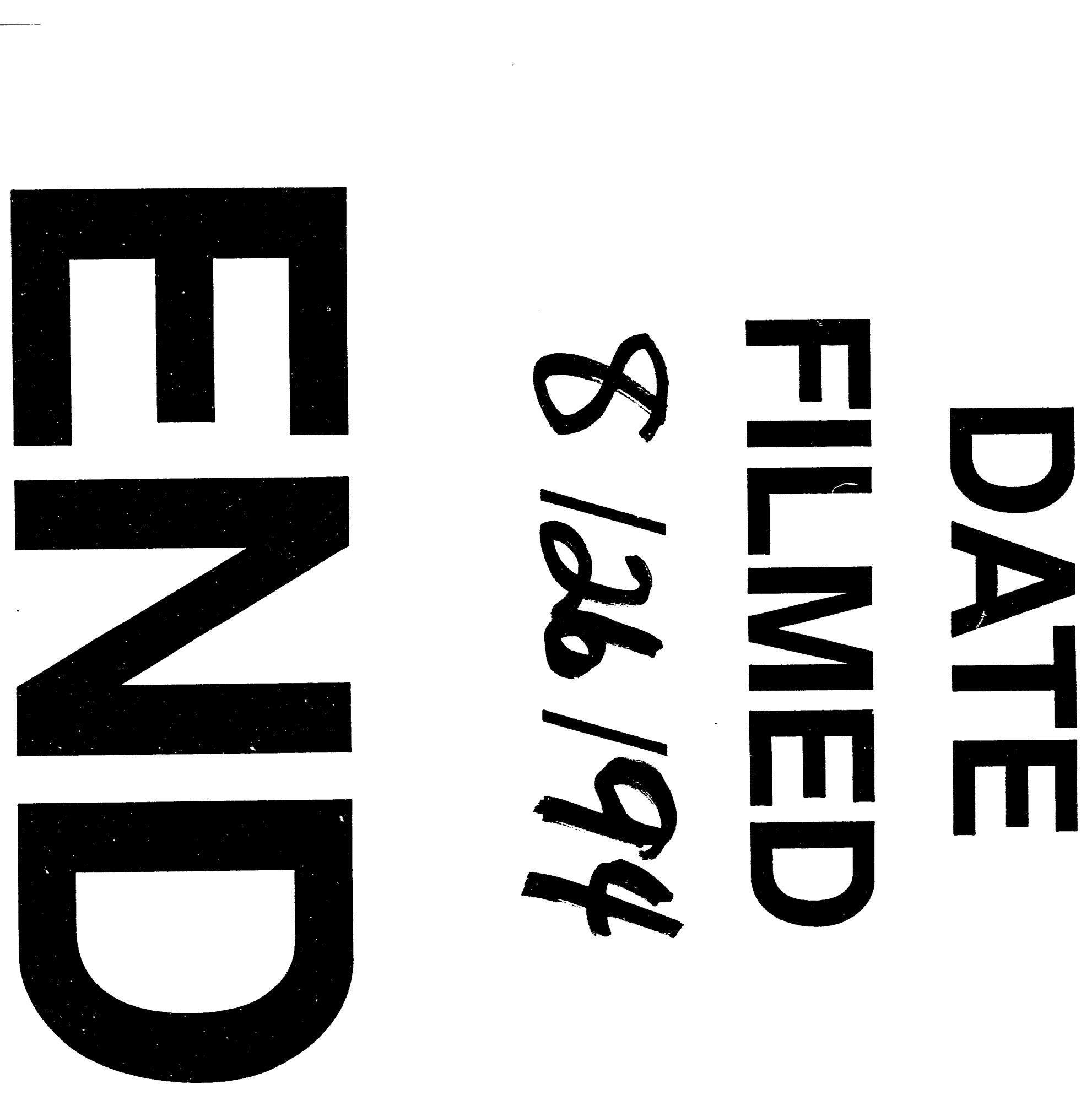
